\title{
THE SPATIAL VECTORCARDIOGRAM IN HYPOTHERMIA
}

\author{
BY \\ D. EMSLIE-SMITH * \\ From the Baker Medical Research Institute, and Clinical Research Unit, Alfred Hospital, Melbourne, Australia \\ Received August 8, 1957
}

In hypothermia characteristic changes appear in the electrocardiogram of man and other animals. Most striking of these is a slowly inscribed deflection that appears at the junction of the QRS complex and the S-T segment and grows in amplitude and duration as temperature falls (Emslie-Smith, 1956) (Fig. 1). This deflection, which was first described by Grosse-Brockhoff and Schoedel (1943), is not usually seen in all leads. In man some leads may show, instead, a widening of the base of the QRS complex.

In order to investigate the nature of this deflection more fully, spatial vectorcardiogram loops have been constructed for five patients and one dog, before and after cooling. Hypothermia produces consistent changes in the spatial vectorcardiogram unlike any previously described.

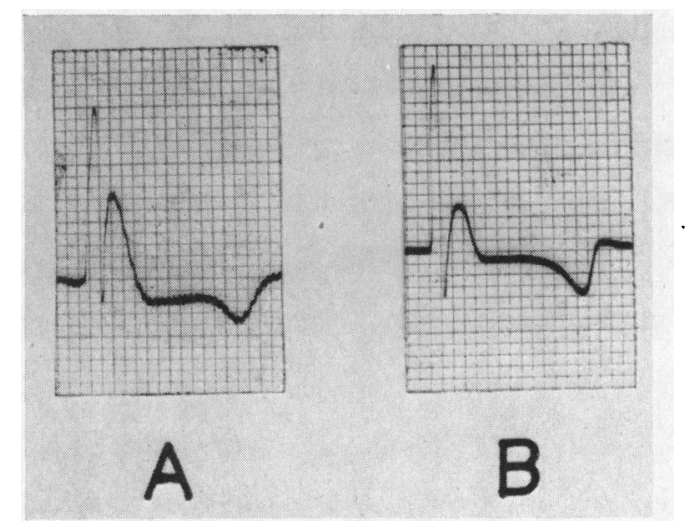

FIG. 1.-(A) Præcordial lead V4 recorded from a man with a normal heart at a temperature of $32^{\circ} \mathrm{C}$. (rectal). (B) Direct electrogram recorded from the epicardium of the left ventricle of a dog at a temperature of $25 \cdot 2^{\circ} \mathrm{C}$. (œsophageal).

\section{MATERIAL AND MethodS}

Each of the five patients had suffered recent subarachnoid hæmorrhage from a congenital cerebral aneurysm. There were four women and one man, their ages ranging from 27 to 49 years. Hypothermia in preparation for craniotomy was produced by the technique of skin-cooling previously described (EmslieSmith, 1956). Rectal temperatures were recorded by a thermistor apparatus. One patient (Case 3 ) had mild diastolic hypertension at the time of observation, but no clinical or electrocardiographic evidence of heart disease.

After the patients were anæsthetized, but before they were cooled, the electrocardiogram was recorded on a direct-writing electrocardiograph (Electrite, Cambridge Instrument Co. Ltd.) using standard and unipolar limb leads, and præcordial leads V1 and V4. It was again recorded after cooling. After complete

* Edward Wilson Memorial Fellow, 1955-56. Present address : Dept. of Medicine, Queen's College, Dundee. 
recovery from the operation simultaneous records of leads aVR and aVL, V1, and V4 (and sometimes also of $\mathrm{V} 4$ and aVL) were made at fast paper-speed on a photographically-recording, mirror-galvanometer electrocardiograph (New Electronic Products Ltd.). At this time direct three-plane vectorcardiograms were also recorded by the method of electronic synthesis described by Lowe and Goble (1952). The projections of the vectorcardiograms in the three planes were those recommended by the American Heart Association (Wilson et al., 1954).

From leads aVR, aVL, V1, and V4 frontal and horizontal vectorcardiograms were graphically derived, using the conventions employed by Lowe and Goble (1952). These were combined, as plan and elevation, to make three-dimensional wire models of the QRS and T spatial vectorcardiogram loops before and after cooling.

The dog was anæsthetized with intravenous pentobarbitone and the temperature was reduced by skin cooling. Electronic three-plane vectorcardiograms were recorded before and during cooling, using a modification of the cuboidal electrode placement of Duchosal and Sulzer (1949). From the records thus obtained three-dimensional wire models were constructed to show the spatial vectorcardiogram before and after cooling.

From the wire models the angles of the axes of the QRS and T loops, and the spatial angle between these axes (QRS-T spatial angle), were measured and recorded according to the conventions of Lowe and Goble (1952).

\section{RESULTS}

In most cases the frontal and horizontal vectorcardiograms (VC) that were graphically derived from scalar leads before cooling resembled fairly closely those obtained by electronic synthesis at normal temperatures after operation. There were sometimes minor differences in the orientation of loops of similar shape (Fig. 5).

At normal temperatures 4 out of 5 of the human vectorcardiograms were within normal limits, and in Case 3 it was suggestive of mild left ventricular hypertrophy, although in the scalar leads the only abnormality was slight flattening of $\mathrm{T}$ in $\mathrm{aVL}$.

Before cooling, the QRS vector loop from the dog was directed more anteriorly than would be normal in man but in other respects it closely resembled a human vectorcardiogram with a QRS loop of semivertical type (Fig. 2 and 3).

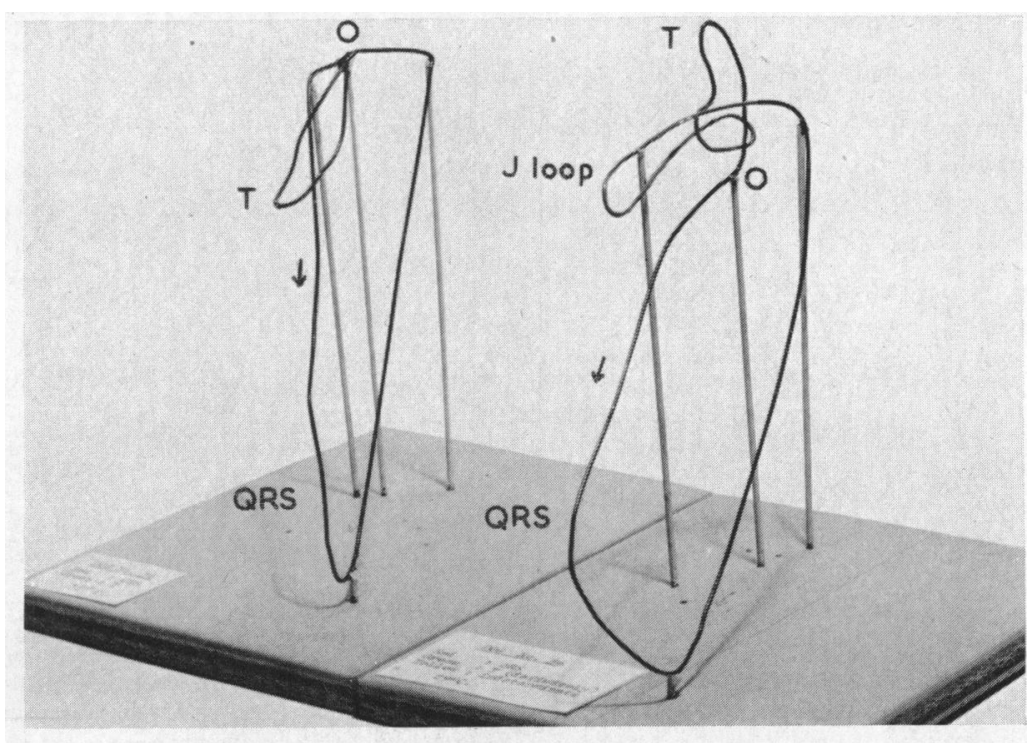

FIG. 2.-Wire models of the electronically synthesized spatial vectorcardiogram loops of dog at œsophageal temperatures of $37^{\circ} \mathrm{C}$. (left) and $29^{\circ} \mathrm{C}$. (right). The P loop is not shown. The loops are viewed from the front, slightly above and to the (model's) left of the origin (O). The arrows denote the direction of inscription of the QRS loops. Note the extra J loop characteristic of hypothermia. 
DOG No 56
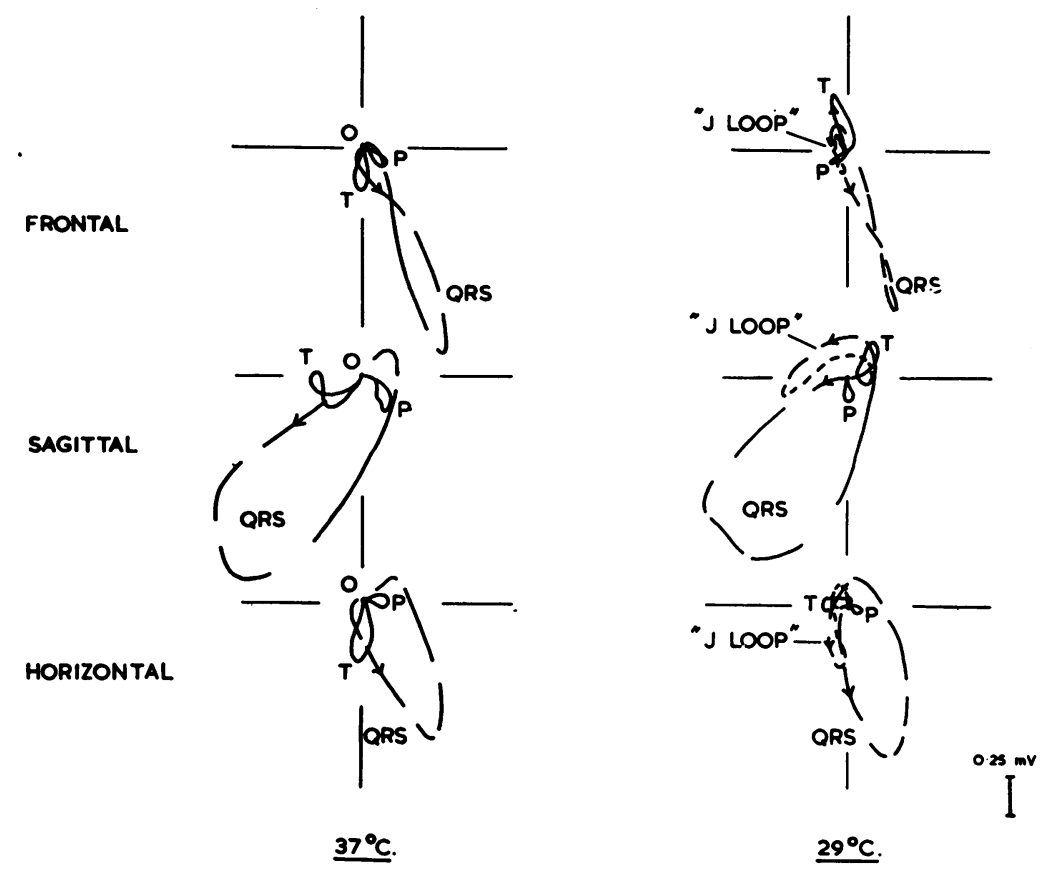

FIG. 3.-The vectorcardiographic loops in three planes (traced from the photographic records) from which the wire models in Fig. 2 were constructed. The inscription of the loops is interrupted, by a blanking pulse, every $0.006 \mathrm{sec}$.

Striking and consistent changes appeared in the vectorcardiograms of all five patients on cooling to $31.5^{\circ} \mathrm{C}$. or lower. The main part of the QRS loop was essentially unchanged, but deformity appeared in each case in the terminal crochet of the QRS loop and in the junction (J) of the QRS and $\mathrm{T}$ loops, and sometimes in the $\mathrm{T}$ loop.

An extra loop appeared between the QRS and T loops, directed forward and to the left in all cases, and also downward in four. In all cases it was inscribed clockwise in the horizontal plane (Fig. 4), and in four cases it involved a J-shift, the QRS loop failing to return to the origin (O). For this reason it may be referred to as the $J$ loop. It was larger than the $T$ loop in two cases (Cases 1 and 4) and in Case 1 its total length in the horizontal plane was little smaller than that of the QRS loop (Fig. 4). The rate of inscription of the J loop was much slower than that of the QRS loop, and it slowed progressively during the course of each inscription, so that it finally approached that of the T loop (Fig. 3). The T loop that followed this extra loop was open, and in Cases 1 and 5 the QRS-T spatial angle was increased.

After cooling the dog to $29^{\circ} \mathrm{C}$. the direct, electronically synthesized vectorcardiogram showed the same features as those of the human subjects, but the $J$ loop was directed forward, slightly down, and slightly to the right. The QRS-T spatial angle was greatly increased, the T loop pointing in a direction almost opposite to its original one (Fig. 2 and 3).

Detailed descriptions of the spatial vectorcardiogram loops of the dog and of one human patient are given below. Details of the J loops and changes in QRS-T spatial angle seen in hypothermia in all five patients, and in the dog, are shown in Table $I$.

\section{Details of Case 4}

Normal Temperature $\left(37.4^{\circ} \mathrm{C}\right.$.). The QRS loop is inscribed clockwise in the frontal plane, and anticlockwise in both sagittal and horizontal planes. It is wide and semivertical. The initial crochet runs up 

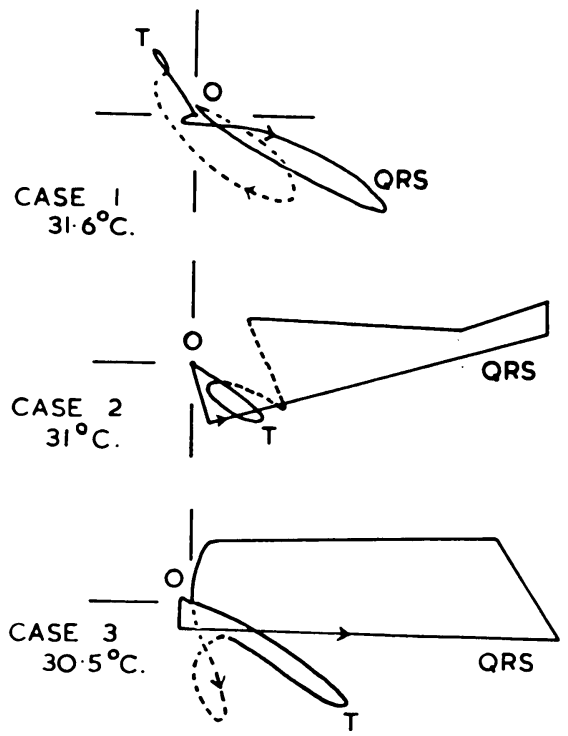

\section{HORIZONTAL}

FIG. 4.-Graphically-derived vectorcardiographic loops in the horizontal plane of all five patients at low temperature. The $\mathbf{J}$ loop is shown as dotted, and is inscribed in a clockwise direction in all cases.

TABLE I

Details of J Loops and Changes in QRS-T Spatial ANgles on Cooling

\begin{tabular}{|c|c|c|c|c|c|}
\hline \multirow{3}{*}{$\begin{array}{l}\text { Case } \\
\text { No. }\end{array}$} & \multirow{3}{*}{$\begin{array}{c}\text { Rectal } \\
\text { temperature } \\
\left({ }^{\circ} \mathrm{C} .\right)\end{array}$} & \multicolumn{3}{|c|}{ J loop } & \multirow{3}{*}{$\begin{array}{c}\text { QRS-T } \\
\text { spatial } \\
\text { angle }\end{array}$} \\
\hline & & \multirow{2}{*}{ Shape } & \multicolumn{2}{|c|}{ Direction of inscription in space } & \\
\hline & & & $\begin{array}{c}\text { Centrifugal } \\
\text { limb }\end{array}$ & $\begin{array}{l}\text { Centripetal } \\
\operatorname{limb}\end{array}$ & \\
\hline \multirow{2}{*}{1} & Normal & & & & $18^{\circ}$ \\
\hline & $31 \cdot 5$ & $\begin{array}{l}\text { Oval } \\
\text { Very large }\end{array}$ & $\mathrm{D}, \mathrm{F}, \mathrm{L}^{*}$ & $\mathrm{U}, \mathrm{B}, \mathrm{R}$ & $130^{\circ}$ \\
\hline \multirow{2}{*}{2} & Normal & & & & $25^{\circ}$ \\
\hline & $31 \cdot 0$ & V-shaped & $\mathrm{D}, \mathrm{F}, \mathrm{l}$ & $\mathrm{U}, \mathrm{F}, \mathrm{R}$ & $25^{\circ}$ \\
\hline \multirow{2}{*}{3} & Normal & & & & $50^{\circ}$ \\
\hline & $30 \cdot 5$ & J-shaped & $\mathrm{d}, \mathrm{F}, \mathrm{L}$ & $\begin{array}{l}\mathrm{U}, \mathrm{B}, \mathrm{r} ; \\
\text { then B,D }\end{array}$ & $35^{\circ}$ \\
\hline \multirow{2}{*}{4} & Normal & & & & $25^{\circ}$ \\
\hline & $30 \cdot 6$ & V-shaped & $\mathrm{D}, \mathrm{F}, \mathrm{l}$ & $\mathrm{U}, \mathrm{b}, \mathrm{r}$ & $15^{\circ}$ \\
\hline \multirow{2}{*}{5} & Normal & & & & $25^{\circ}$ \\
\hline & $31 \cdot 5$ & J-shaped & $\mathrm{U}, \mathrm{F}, \mathrm{l}$ & $\mathrm{D}, \mathrm{B}, \mathrm{R}$ & $40^{\circ}$ \\
\hline \multirow{2}{*}{ Dog } & Normal & & & & $25^{\circ}$ \\
\hline & $29 \cdot 0$ & See Fig. 2 & $\mathrm{~d}, \mathrm{~F}, \mathrm{R}$ & $\begin{array}{l}\mathrm{u}, \mathrm{B}, \mathrm{r} \text {; then } \\
\text { curls to } \mathrm{L} \\
\text { and } \mathrm{R}\end{array}$ & $150^{\circ}$ \\
\hline
\end{tabular}

${ }^{*} \mathrm{D}=$ down, $\mathrm{U}=$ up; $\mathrm{F}=$ forward, $\mathrm{B}=$ back; $\mathrm{L}=$ to the left, $\mathrm{R}=$ to the right. Small letters imply only a small change in direction, e.g. $d=$ slightly down. 
slightly to the right and slightly back. The centrifugal limb runs to the left, slightly down and slightly forward, then it turns steeply down, slightly forward and slightly to the left. The centripetal limb runs back, up and to the right, then turns forward, up and to the right, to $O$. The QRS axis angles are $60^{\circ}$ in the frontal, $110^{\circ}$ in the sagittal, and $20^{\circ}$ in the horizontal plane. The T loop is oval, with its apex slightly below, to the left, and in front of $O$. Its axis angles are $35^{\circ}$ in the frontal, $125^{\circ}$ in the sagittal, and $25^{\circ}$ in the horizontal, plane. The QRS-T spatial angle is $25^{\circ}$. This is a normal, derived VC (Fig. 5 and 6).

After Cooling $\left(30 \cdot 6^{\circ} \mathrm{C}\right.$.). The QRS loop of the derived vectorcardiogram is inscribed clockwise in the frontal, and anticlockwise in the sagittal and in the horizontal, plane. The initial crochet runs up and slightly to the right. The centrifugal limb runs to the left and slightly forward, turns steeply down to the left and slightly forward, and then curves back and slightly to the right. The centripetal limb runs down and back to the right; then it turns sharply up to the right and slightly back, to a point to the left of $O$, where the $\mathrm{J}$ loop tegins. The QRS axis angles are $50^{\circ}$ in the frontal, $100^{\circ}$ in the sagittal, and $20^{\circ}$ in the horizontal plane. The J loop is V-shaped. Its centrifugal limb runs down, forward, and slightly to the left; its centripetal limb runs up, slightly back and to the right, to a point slightly above, to the right, and slightly in front of $\mathrm{O}$, where the $\mathrm{T}$ loop begins. The open $\mathrm{T}$ loop is narrow and twisted, with its apex below, to the left and in front of $O$. Its axis angles are $30^{\circ}$ in the frontal, $140^{\circ}$ in the sagittal and $30^{\circ}$ in the horizontal, plane. The QRS-T spatial angle is $15^{\circ}$ (Fig. 5 and 6).

\section{Details of A DoG}

Normal Temperature $\left(37^{\circ} \mathrm{C}\right.$.). The electronically synthesized QRS loop is inscribed in a figure of eight in the frontal plane, and anticlockwise in both sagittal and horizontal planes. Its position is semivertical. The initial crochet runs forward, to the right and slightly down. The centrifugal limb runs to the left,

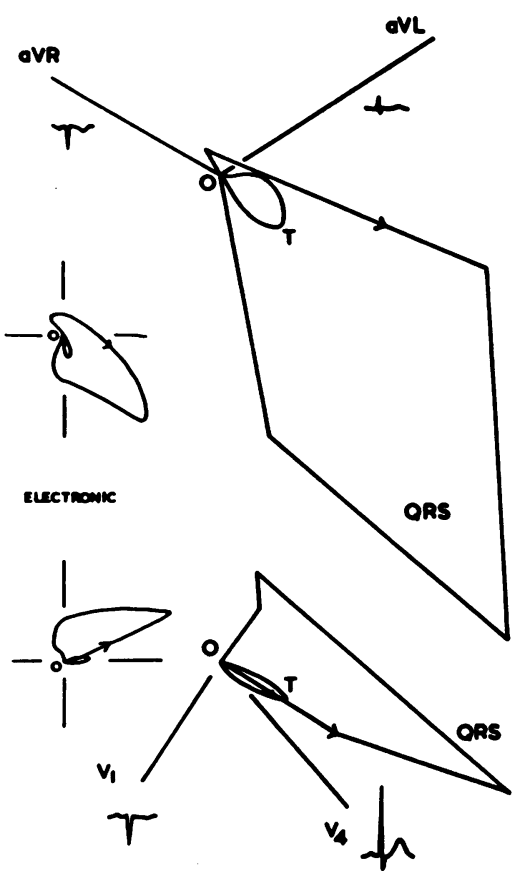

$37 \cdot 4^{\circ} \mathrm{C}$

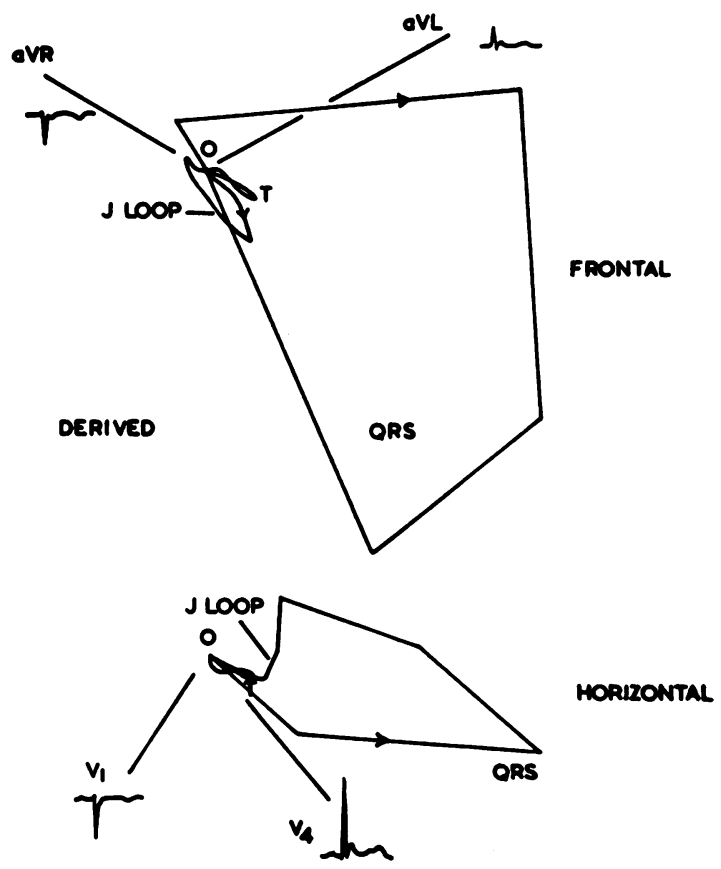

$30.5^{\bullet} \mathrm{C}$.

FIG. 5.-Frontal and horizontal QRS and $\mathrm{T}$ loops at $37.4^{\circ}$ and $30.5^{\circ} \mathrm{C}$. in Case 4 . The large vectorcardiograms were graphically derived from the scalar lead complexes shown. The small ones were electronically recorded at normal temperature. Apart from a difference in orientation in the horizontal plane the graphic and electronic loops resemble one another. At $30 \cdot 5^{\circ} \mathrm{C}$. the J loop has appeared. 
down and forward; the centripetal limb runs up, back and to the right, to a point behind and to the left of $O$, then in to $O$. The QRS axis angles are $65^{\circ}$ in the frontal, $125^{\circ}$ in the sagittal, and $55^{\circ}$ in the horizontal plane. The $\mathrm{T}$ loop is narrow, directed forward and down from $\mathrm{O}$. Its axis angles are $90^{\circ}$ in the frontal, $140^{\circ}$ in the sagittal, and $85^{\circ}$ in the horizontal plane. The QRS-T spatial angle is $25^{\circ}$. Judged by human criteria the QRS and T axis angles are too large in the horizontal, and the T axis angle too large in the sagittal, plane: that is, the QRS loop is directed too anteriorly. Apart from this the vectorcardiogram resembles that from a normal man (Fig. 2 and 3).

After Cooling $\left(29^{\circ} \mathrm{C}\right.$.). The electronically synthesized QRS loop is inscribed in a figure of eight in the frontal plane, and anticlockwise in the sagittal and horizontal planes. Its position is unchanged. The initial crochet is absent, the centrifugal limb running forward to the left and down. The centripetal limb runs up and almost straight back to a point behind, slightly above and slightly to the left of $O$, where the $\mathrm{J}$ loop begins. The QRS axis angles are $65^{\circ}$ in the frontal, $135^{\circ}$ in the sagittal, and $75^{\circ}$ in the horizontal plane. The $\mathbf{J}$ loop is conspicuous. Its centrifugal limb runs forward, to the right and slightly down. Its centripetal limb runs back, slightly up and slightly to the right; then it curls down to the left, and up again to the right to start the $\mathrm{T}$ loop. The $\mathrm{T}$ loop is $\mathrm{U}$-shaped. Its centrifugal limb runs up, slightly to the right and slightly back; its centripetal limb runs down, slightly to the left and forward to $O$. The $T$ axis angles are $-110^{\circ}$ in the frontal, $70^{\circ}$ in the sagittal and $-140^{\circ}$ in the horizontal, plane. The QRS-T spatial angle has increased to $150^{\circ}$ (Fig. 2 and 3).

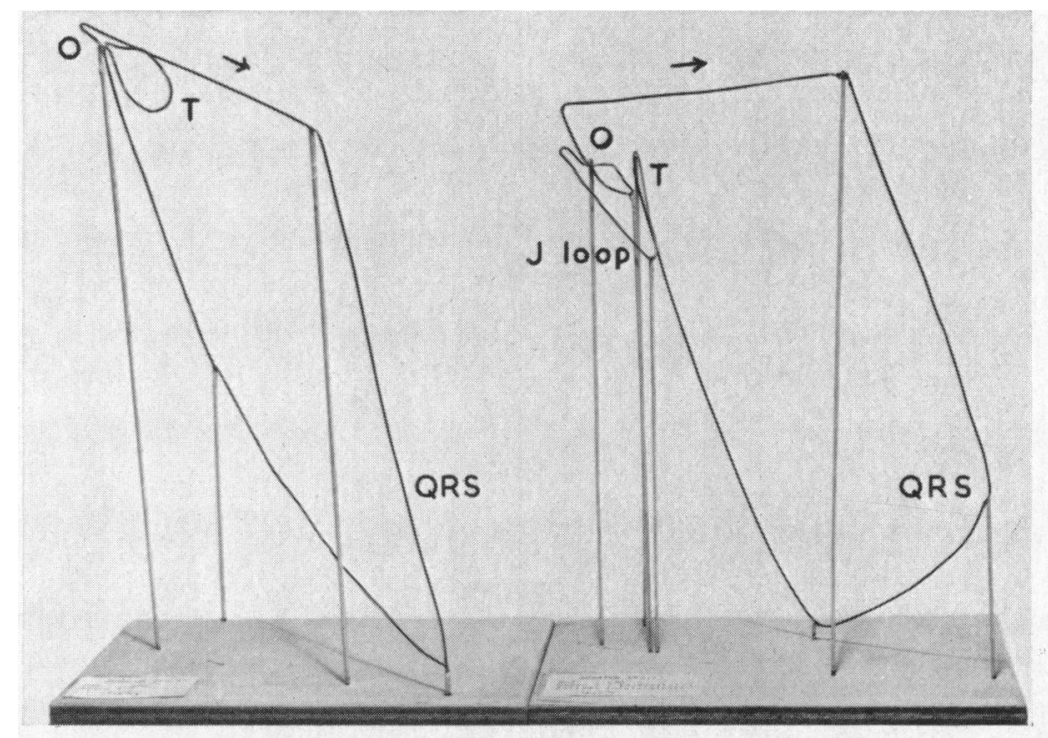

FIG. 6.-Wire models of the spatial vectorcardiographic loops (QRS and T) of Case 4 at $37 \cdot 4^{\circ} \mathrm{C}$. (left) and $30 \cdot 5^{\circ} \mathrm{C}$. (right). The loops are viewed from the front and slightly above. Arrows denote the direction of inscription of the QRS vector loops.

\section{DisCUSSION}

Vectorcardiograms derived from scalar leads by graphic methods are known to be considerably less accurate than those directly obtained by electronic synthesis (Sayers, 1952), particularly if the scalar tracings used for the construction of a loop in any one plane are not recorded simultaneously. In this study it was impossible to use the bulky and relatively fixed vectorcardiograph apparatus in the neurosurgical operating theatre, and non-simultaneous directly-written scalar tracings had to be used. However, by later recording the relevant scalar tracings simultaneously on a photographically-recording electrocardiograph, simultaneous points of reference could be identified on each of the paired tracings recorded in the theatre. In this manner errors due to phase-shift should be largely eliminated. As a further check the frontal and horizontal vectorcardiograms 
thus graphically derived at normal temperatures were compared with those later recorded directly from the same patient by electronic synthesis. These precautions suggest that apart from some discrepancies of orientation, perhaps due to posture and the effects of anæsthesia, the derived vectorcardiograms probably resemble those that would have been obtained electronically. This belief is strengthened by the fact that the vectorcardiogram of the dog, recorded directly by electronic means throughout cooling, showed essentially the same change as the loops graphically derived from the patients.

This appears to be the first study of the effect of hypothermia on the vectorcardiogram. It demonstrates that cooling produces gross consistent changes in the spatial vectorcardiogram of man and dog. The characteristic change during hypothermia is a disturbance involving the terminal crochet of the QRS loop, the junction (J) of the QRS and T loops, and the T loop. The last part of the centripetal limb of the QRS loop is suddenly deflected forward and greatly slowed, so that the QRS loop does not always return to the point of origin (J-shift) but continues, to form an extra, conspicuous open loop. This, in turn, continues into a more or less U-shaped T loop which may lie in a direction different from that seen at normal temperature (Fig. 2).

This striking $\mathbf{J}$ loop is unlike anything seen in left or right bundle-branch block (Lowe and Gardiner, 1954) or ventricular hypertrophy (Gardiner and Lowe, 1953 and 1954). Spatially it appears to begin before the QRS loop is ended, but in rate of inscription it more resembles the $T$ loop, with which it is directly continuous. In some cases as cooling proceeds there is a progressive increase in the QRS-T spatial angle, and at low temperatures the long axis of the T loop may lie almost opposite in direction to the QRS loop. When this occurs the $J$ and $T$ loops may form a more or less continuous loop as large as the QRS loop. The fact that changes also appear in the $\mathrm{T}$ loop does not necessarily imply that they are causally related to the development of the $\mathbf{J}$ loop, for both changes might arise independently, from the effects of hypothermia.

From this study alone it is impossible to draw any conclusion about whether hypothermia primarily disturbs the process of depolarization or of repolarization, or both. The new J loop involves both the terminal crochet of the QRS loop and the first part of the T loop. Its rate of inscription progressively slows, to approach that of the T loop (Fig. 3). The work of Burgen and Terroux (1953) and of Coraboeuf and Weidmann (1954) has shown that cooling slows the processes of depolarization and repolarization of the single myocardial fibre at different rates. This implies that in the intact heart the overall relationship between the processes of depolarization and repolarization is altered in hypothermia. An alteration of this nature could be expected to modify the total unbalanced electrical activity of the heart during the part of the cycle when depolarization is normally ending and repolarization is beginning. This is precisely the part of the cycle occupied by the characteristic scalar deflection of hypothermia and the $\mathbf{J}$ loop of the vectorcardiogram. Since the electrocardiogram represents the total unbalanced electrical activity of the heart, manifest in terms of potential difference at the site of the electrode, the $J$ loop of the vectorcardiogram and the characteristic deflection in scalar leads may reflect the altered relationship between depolarization and repolarization that is produced by hypothermia.

\section{SUMMARY}

From scalar-lead electrocardiograms of five patients without heart disease, vectorcardiograms in the frontal and horizontal planes were graphically derived for normal and low temperatures. Wire models of the spatial vectorcardiograms were constructed.

In hypothermia the main part of the QRS loop was essentially unchanged but below $31.5^{\circ} \mathrm{C}$. consistent alterations appeared in the terminal crochet of the QRS loop, in the junction (J) of QRS and $\mathrm{T}$ loops, and in the T loop. An extra loop ( $\mathrm{J}$ loop) appeared between the QRS and T loops, inscribed clockwise in the horizontal plane and directed forward, to the left, and usually downward. Its rate of inscription slowed progressively during the course of each inscription, so that finally it approached that of the T loop. The spatial angle between the QRS and T loop sometimes increased greatly. 
Three-plane electronic vectorcardiograms were made during the cooling of one dog. The spatial vectorcardiogram at $29^{\circ} \mathrm{C}$. showed an increase in the QRS-T angle and a $\mathrm{J}$ loop similar to those derived from the human subjects.

The J loop may represent an altered relationship between the overall processes of depolarization and repolarization in hypothermia.

I should like to thank Mr. K. C. Bradley and Dr. R. H. Orton for permission to study and report these cases, Dr. T. E. Lowe (Director of the Baker Medical Research Institute and Alfred Hospital Clinical Research Unit) for his help and encouragement, and Professor I. G. W. Hill for his help in the preparation of the manuscript. I thank the Editor, Australasian Annals of Medicine, for permission to reproduce Fig. 1.

\section{REFERENCES}

Burgen, A. S. V., and Terroux, K. G. (1953). J. Physiol., 119, 139.

Coraboeuf, E., and Weidmann, S. (1954). Helv. physiol. pharmacol. Acta., 12, 32.

Duchosal, P. W., and Sulzer, R. (1949). La Vectocardiographie. Basle, S. Karger.

Emslie-Smith, D. (1956). Aust. Ann. Med., 5, 62.

Gardiner, J. M., and Lowe, T. E. (1953). Aust. Ann. Med., 2, 22.

- - (1954). Aust. Ann. Med., 3, 18.

Grosse-Brockhoff, F., and Schoedel,W. (1943). Arch. Exper. Path. Pharmakol., 201, 417.

Lowe, T. E., and Goble, A. J. (1952). Med. J. Aust., 1, 503.

-, and Gardiner, J. M. (1954). Aust. Ann. Med., 3, 289.

Sayers, B. McA. (1952). Aust. Ann. Med., 1, 154.

Wilson, F. N., Kossmann, C. E., Burch, G. F., Goldberger, E., Graybiel, A., Hecht, H. H., Johnston, F. D., Lepeschkin, E., and Myers, G. B. (1954). Circulation, 10, 564. 